\title{
In-Situ TEM Observations of Carbon Nanotube Growth By The Catalytic Decomposition of Acetylene
}

\author{
M. M. J. Treacy ${ }^{*}$, M. Brown ${ }^{*}$, P. Rez ${ }^{*}$, G.H. Du ${ }^{* *}$ and R. Sharma** \\ *Dept. of Physics and Astronomy, Arizona State University, Tempe, AZ 85287 \\ ${ }^{* *}$ Center for Solid State Science, Arizona State University, Tempe, AZ 85287
}

Carbon nanotubes are known to have special mechanical and electrical properties [1]. These properties have created a number of possible applications ranging from the nanoscale to the mesoscale. Consequently, much research is dedicated to understanding their physical properties and how they are affected by the precise structure of the nanotubes. Modeling shows that the electrical properties of a single nanotube shell should depend sensitively on the nanotube structure; they could be either conducting or semiconducting depending on their chirality and physical diameter [2]. Nanotube stiffness and yield strength is less sensitive to the details of perfect structures, but is strongly affected by defects. When it was discovered how to make large quantities of single-walled nanotubes with different diameters and chiralities, the possibility of exploiting the electrical properties became closer to practical realization [3].

In order to exploit their structure-dependent properties, it is important to be able to control nanotube synthesis. Several techniques have now been discovered for making single-walled nanotubes. Laser sputtering of a graphite target makes ropes of straight single-walled nanotubes, which can be later separated into single nanotubes [3]. Catalytic decomposition of acetylene by finely dispersed particles, such as $\mathrm{Ni}, \mathrm{Au}$ and $\mathrm{Fe}$ on inert silica supports, can also make single-walled nanotubes at considerably low cost. However, such catalytically synthesized nanotubes are not always straight, tending to be curved with irregular diameters and structures that vary with the synthesis conditions. The defects that cause such structural irregularities must also affect the electrical properties. To successfully exploit the electrical properties of carbon nanotubes, it is important that their synthesis be reproducible, with control over the defects. To understand the growth mechanisms of catalytically grown single-walled carbon nanotubes, we are studying their formation in-situ in the TEM.

We have observed carbon nanotube growth in-situ in a specially modified Tecnai F-20 [4]. Observations are made at temperatures ranging from $450^{\circ} \mathrm{C}$ to $600^{\circ} \mathrm{C}$ and pressures ranging from 1 to $16 \mathrm{mT}$ Torr of acetylene, $\mathrm{C}_{2} \mathrm{H}_{2}$. Carbon nanotubes were observed to grow by different mechanisms depending upon the growth conditions. For example, Figure 1 shows single-walled nanotubes. These tubes are notable for the extended straight sections, although many curved sections are present. In some experiments, nanotubes are tightly curved and the tangle can resemble a (self-avoiding) random walk. The growth rate can be estimated directly from video frames (Figure 2a). Under some growth conditions, the growth speed of nanotubes, projected onto the image varies according to a 2dimensional Maxwellian speed distribution (Figure 2b). In other words, the nanotube tip growth resembles the stochastic behavior of a gas molecule! Under other growth conditions (i.e. temperature, $\mathrm{C}_{2} \mathrm{H}_{2}$ pressure, and possibly catalyst particle size and habit) the nanotube grows fairly straight and its growth rate is less random.

It is expected that in-situ TEM observations of nanotube formation, as a function of temperature, pressure and catalyst structure, will reveal the growth mechanisms at low pressures. 

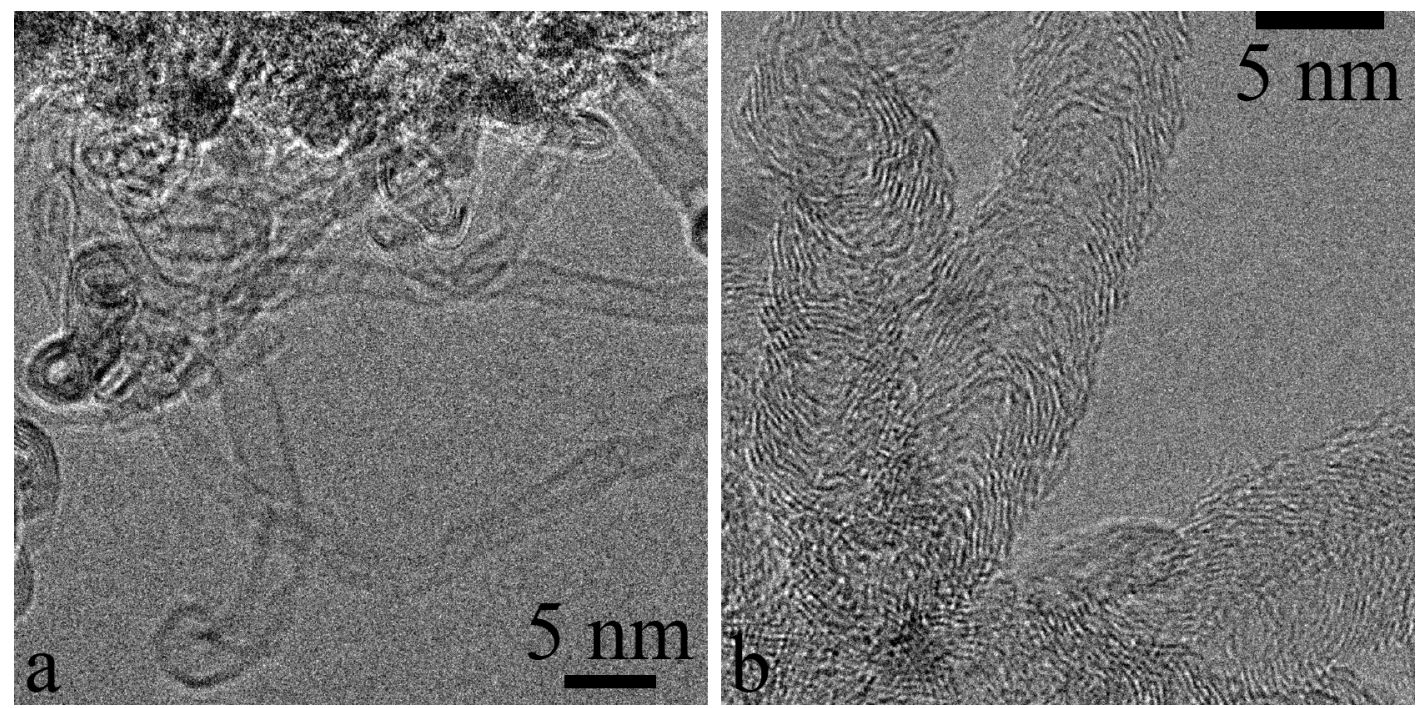

Fig. 1. a) Single-walled carbon nanotubes grown in-situ under observation. These nanotubes have extended straight sections. b) By changing the synthesis conditions, the nanotube structure can change radically. In this instance, filaments comprising stacked nano-cones have grown.
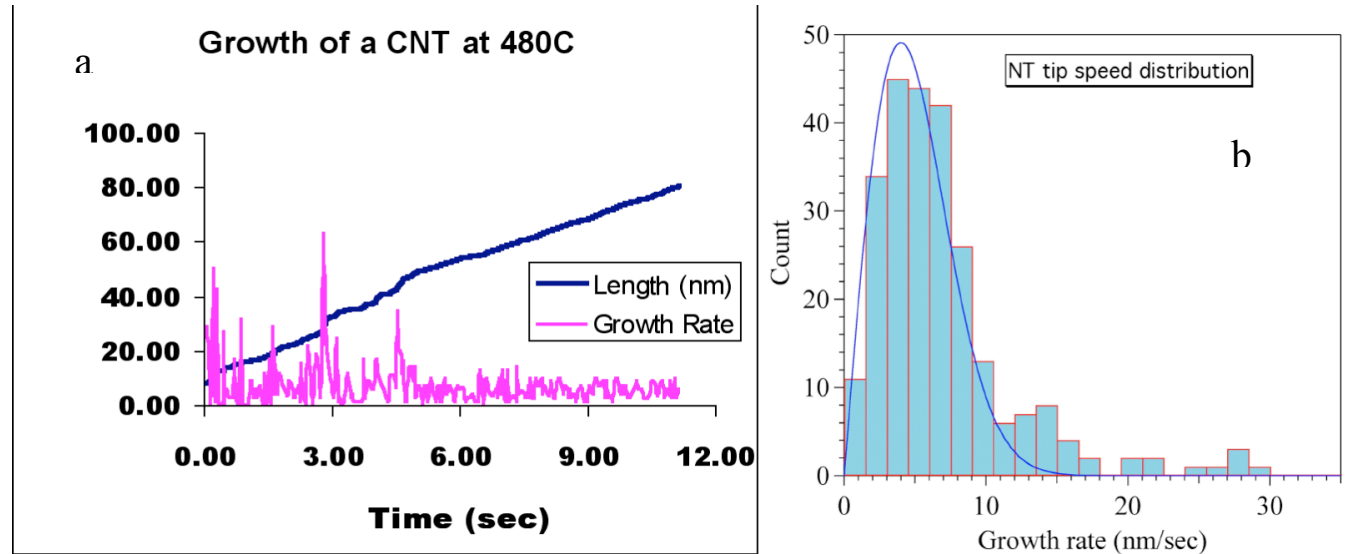

Fig. 2. a) Experimental plot showing the projected length and tip growth speed of a nanotube as a function of time. b) Histogram of the 2-D tip growth speeds. The speeds are distributed in a manner reminiscent of a 2-dimensional Maxwellian speed distribution (continuous line). There is a secondary peak at $15 \mathrm{~nm} / \mathrm{sec}$, which appears to correspond to growth spurts of straight nanotube sections.

\section{References}

[1] Iijima, S. Nature 354 (1991) 56-58.

[2] Dekker, C., Phys. Today, (1999) May, 22-28.

[3] Guo, T., Nikolaev, P., Rinzler, A.G., Tom'anek, D., Colbert, D.T., Smalley, R.E.: J. Phys. Chem. 99 (1995) 10694.

[4] Sharma, R., Rez, P., Treacy, M.M.J., and Stuart, S.J., J. Electron Microsc. 54 (2005) 231.

[5] The funding from NSF-CTS\#0508434 and the use of John Cowley Center for High Resolution Electron Microscopy is gratefully acknowledged. 